\section{Fatores associados à realização da prevenção secundária do câncer de mama no Município de Maringá, Paraná, Brasil}

\author{
Factors associated with secondary breast cancer \\ prevention in Maringá, Paraná State, Brazil
}

\author{
1 Unidade de Ensino Superior \\ Ingá, Faculdade Ingá, \\ Maringá, Brasil. \\ 2 Universidade Estadual de \\ Maringá, Maringá, Brasil. \\ Correspondência \\ J. C. Matos \\ Unidade de Ensino Superior \\ Ingá, Faculdade Ingá. \\ $A v$. Colombo 9727, Maringá, \\ PR 87070-810, Brasil. \\ jessicamaringa@yahoo.com.br
}

\begin{abstract}
This study analyzed the prevalence of secondary breast cancer prevention and associated factors in women 40 to 69 years of age in Maringá, Paraná State, Brazil. Prevalence was $64.5 \%$ for breast self-examination, $71.5 \%$ for clinical examination, and 79\% for mammography. The data suggest that age, schooling, ethnicity, socioeconomic status, religion, and prior hormone replacement therapy influenced the rates of the three types of examination. Preventive practices were significantly more common among women of higher socioeconomic status. Proper orientation and performance of preventive measures and tests should be at the reach of all women in the highest-risk age bracket, thereby decreasing late diagnosis and avoidable deaths and increasing the odds of curing breast cancer.
\end{abstract}

Breast Neoplasms; Secondary Prevention; Early Diagnosis; Prevalence
Jéssica Carvalho de Matos 1

Sandra Marisa Pelloso 2

Maria Dalva de Barros Carvalho 2

\section{Introdução}

As neoplasias apresentam-se como uma das principais causas de morbidade e mortalidade na população mundial, e, entre as mulheres, destaca-se o câncer de mama. Estima-se que, a cada ano, ocorram mais de 1.050 .000 casos novos de câncer de mama em todo o mundo, o que torna esse tipo de câncer o mais comum entre as mulheres 1 .

No ano de 2006, foi estimado em 48.930 o número de novos casos de câncer de mama no Brasil, correspondendo a 51,66 casos por $100 \mathrm{mil}$ mulheres 2 . A estimativa de 2010 é de 49.400 casos novos no Brasil, com risco estimado de 49/100 mil mulheres 3 .

No Estado do Paraná, essa realidade demonstra-se preocupante e pode ser constatada pela estimativa de 2.970 casos novos de câncer de mama (56,7/100 mil mulheres) no ano de 2006. No ano de 2008, a estimativa foi de 56,16 casos para cada 100 mil mulheres. Em 2010, estimouse 2.990 casos de câncer de mama $(54,4 / 100$ mil mulheres) ${ }^{4}$. Observa-se que a estimativa de casos novos de câncer de mama no Paraná é maior que a estimativa nacional.

Na Região Sul, com exceção dos tumores de pele, o câncer de mama é o mais frequente entre as mulheres, perfazendo um coeficiente de 69/100 mil mulheres. Na Região Sudeste, esse coeficiente é de 65/100 mil mulheres. Nas demais regiões, essa estatística é menor 4 . 
Dentro desse contexto, é necessário ressaltar as estatísticas de mortalidade para o Estado do Paraná que, nos anos de 2006, 2007 e 2008, foram de, respectivamente, 630, 642 e 684 mortes. Pode-se afirmar que os óbitos ocorreram, em sua maioria, em mulheres com idade entre 40 e 69 anos (Sistema de Informação sobre Mortalidade. Banco de dados do SUS. http://tabnet. datasus.gov.br/cgi/tabcgi.exe?sim/cnv/obt10pr. def, acessado em 10/Nov/2010).

Em Maringá, os cânceres constituíram a segunda principal causa de mortalidade de mulheres no ano de 2005. Do total de 675 óbitos, 133 foram causados pela doença 5 . Identificaram-se 22 mortes por câncer de mama no ano de 2006 e 22 e 20 mortes no ano de 2007 e 2008, respectivamente (Sistema de Informação sobre Mortalidade. Banco de dados do SUS. http://tabnet. datasus.gov.br/cgi/tabcgi.exe?sim/cnv/obt10pr. def, acessado em 10/Nov/2010).

Embora o aumento na taxa de mortalidade por câncer de mama possa também ser reflexo da melhora das técnicas de diagnóstico e do registro nas declarações de óbito, a principal causa é revelada pela deficiência nas medidas de rastreamento e controle da doença 6 .

A prevenção do câncer de mama é uma questão complexa, devido à multiplicidade de fatores envolvidos que tornam difícil o controle da doença. No caso do câncer de mama, a prevenção secundária se refere ao diagnóstico e tratamento precoce 7 .

Entretanto, as formas mais eficazes para a detecção precoce do câncer de mama são o exame clínico da mama e a mamografia. Atualmente, o autoexame das mamas não é estimulado como estratégia isolada para a detecção precoce, e sim recomendado como ação de educação para saúde que contempla o conhecimento do próprio corpo 8 .

Dentre os métodos para o rastreamento do câncer de mama, a mamografia é considerada o principal método diagnóstico do câncer em estágio inicial, por possibilitar a detecção de alterações ainda não palpáveis, favorecendo um tratamento mais efetivo e menos agressivo 9 .

O câncer de mama, na maioria das vezes, possui um bom prognóstico quando diagnosticado e tratado oportunamente, porém as taxas de mortalidade permanecem elevadas no Brasil, provavelmente pelo fato de a doença ser diagnosticada em estágios avançados. A sobrevida média, após cinco anos, é de $61 \%$ na população mundial 4.

Diante das particularidades em relação ao tema, este estudo teve como objetivo analisar a prevalência e os fatores associados à realização de ações de prevenção secundária do câncer de mama entre mulheres de 40-69 anos do Município de Maringá, Paraná.

\section{Material e métodos}

Foi realizado um estudo analítico, de corte transversal, tipo inquérito populacional domiciliar, no Município de Maringá, Paraná. Os resultados obtidos fazem parte da dissertação de mestrado Prevalência e Fatores Associados à Prevenção Secundária do Câncer de Mama em Maringá- $P R$, apresentada à Universidade Estadual de Maringá 10 .

De acordo com o último recenseamento, a população feminina maringaense, entre 40 a 69 anos, perfaz um total de 48.421 mulheres. Considerando um intervalo de $95 \%$ de confiança (IC95\%; $z=1,96$ ), a estimativa da amostra deverá estar dentro de $\pm 3 \%$ (e) da real proporção da população de mulheres nessa faixa etária. Para tanto, foi utilizado o modelo estatístico $n=z^{2}[p(1-$ p)]: $\mathrm{e}^{2}$ para populações infinitas $(\mathrm{N}>10.000)$, que fornece o tamanho da amostra que, nesse caso, é de 384 mulheres. Com um acréscimo de 14\%, para possíveis perdas, o tamanho total da amostra é de 439 mulheres.

Enfim, o estudo foi realizado com uma amostra de 439 mulheres na faixa etária de 40-69 anos.

A amostragem foi aleatória, estratificada, em que todos os bairros, jardins e zonas do Município de Maringá foram geograficamente colocados em cinco estratos: norte, sul, leste, oeste e centro. Em seguida, foram selecionados os setores que participaram de cada estrato, conforme a densidade demográfica de cada um.

De cada estrato, foi selecionada uma amostra aleatória simples, proporcional ao número de mulheres residentes em cada um desses estratos, tendo em vista o tamanho da amostra (439). Todos os bairros pertencentes a cada estrato foram contemplados com representantes amostrais. No total, foram selecionados 31 bairros do Município de Maringá.

Os dados foram coletados mediante entrevistas domiciliares, com questionário estruturado e pré-testado, contendo questões referentes a aspectos socioeconômicos, biológicos, comportamentais e de utilização de serviços, nos meses de janeiro a março de 2008.

Os dados foram armazenados em planilha do programa Excel e analisados no programa SAS, versão 9.1 (SAS Inst., Cary, Estados Unidos), logo após sua coleta nas entrevistas, com posterior análise com testes estatísticos apropriados.

Foram realizadas a análise univariada (quiquadrado e odds ratio bruta) e a análise multivariada (regressão logística), que estuda a associa- 
ção entre as variáveis desfecho (autoexame das mamas, exame clínico da mama e mamografia) com os fatores em estudo. As variáveis que, na análise univariada, mostraram-se associadas com a realização da prevenção secundária do câncer de mama, com nível de significância observado menor ou igual a $20 \%$ no teste do qui-quadrado ( $\mathrm{p} \leq 0,20$ ), foram selecionadas para a análise multivariada por meio de regressão logística.

Para as mulheres selecionadas que aceitaram participar da pesquisa, foi realizada a leitura do Termo de Consentimento Livre e Esclarecido, redigido de acordo com a Resolução $n^{\circ}$. 196/96 do Conselho Nacional de Saúde, após a qual elas o assinaram.

O projeto foi apreciado e aprovado pelo Comitê de Ética em Pesquisa da Universidade Estadual de Maringá (parecer no. 406/2007).

\section{Resultados}

\section{Caracterização das entrevistadas}

A média de idade das mulheres estudadas foi de 52,84 anos, sendo a raça branca predominante com mais de $80 \%$ (356) das mulheres, e a maioria não possuía trabalho remunerado no período $(62 \%)$.

A classe econômica mais prevalente foi classificada como C (241), seguida da D e B2. Dessas mulheres, 33,9\% (149) possuíam apenas o primário completo, e somente $7,7 \%$ (34) das mulheres possuíam superior completo.

Em sua maioria (295), eram da religião católica, casadas ou moravam junto com seu companheiro (302) (Tabela 1).

\section{Prevalência das condutas preventivas do câncer de mama}

Quanto à prevalência das condutas de prevenção secundária do câncer de mama, entende-se o autoexame das mamas, exame clínico das mamas e mamografia.

A prevalência encontrada do hábito de realizar o autoexame das mamas foi de 64,5\% (283), e, dessas, 76,7\% (217) realizaram o autoexame, ao menos, uma vez ao mês nos últimos seis meses anteriores à pesquisa. Foi orientada pelo profissional de saúde na última consulta ginecológica a realização do autoexame das mamas em 69,7\% (306) dos casos. A prevalência de exame clínico das mamas na última consulta ginecológica foi de $71,5 \%$ (314).

Quanto à prevalência de mamografia, 79\% (347) relataram já ter realizado esse exame alguma vez na vida, ao passo que $84,4 \%$ (293) dessas mulheres realizaram a última mamografia há dois anos ou menos (Tabela 2).

\section{Fatores associados à realização do autoexame das mamas}

Obteve-se que o único fator associado à realização do autoexame das mamas foi a classe econômica da mulher.

Observou-se uma forte correlação entre a classe econômica e a realização do autoexame das mamas. As mulheres de classe econômica C, tanto na análise bruta quanto na ajustada, foram as que mais relataram realizar o autoexame das mamas, já a classe B e as classes C e D se comportaram da mesma maneira $(\mathrm{p}=0,0325)$ (Tabela 3).

\section{Fatores associados à realização do exame clínico das mamas}

As variáveis independentes que estiveram associadas à realização do exame clínico das mamas em Maringá foram: classe econômica, escolaridade e realização de tratamento para menopausa.

$\mathrm{Na}$ análise univariada, observa-se uma tendência linear estatisticamente significativa ( $\mathrm{p}=$ 0,0044) em relação à classe econômica, porém, na análise bruta e ajustada, essa variável não pode ser considerada significativa (Tabela 4).

A população pesquisada de mulheres analfabetas foi aquela que teve menos as suas mamas examinadas clinicamente $(\mathrm{p}=0,0005)$, entretanto, nas outras faixas de escolaridade, não se observou uma correlação com a variável desfecho (Tabela 4).

As mulheres que fizeram, mas já interromperam o tratamento para menopausa são as que mais tiveram suas mamas examinadas clinicamente $(\mathrm{p}=0,0491)$ (Tabela 4).

\section{Fatores associados à realização da mamografia}

Os fatores faixa etária, raça, classe econômica e realização de tratamento para menopausa são fatores que estiveram associados à realização da mamografia.

As mulheres com idade entre 51 a 60 anos foi o grupo que menos fez mamografia $(p=0,0001)$ (Tabela 5). Nas demais faixas etárias, não houve essa correlação.

Quando realizada a análise univariada, observa-se uma correlação entre a raça e a variável desfecho, porém, na regressão logística, não é encontrada essa associação.

A população pesquisada pertencente à camada social mais baixa (classe econômica D e E) é 
Distribuição percentual das entrevistadas segundo variáveis sociodemográficas. Maringá, Paraná, Brasil, 2008.

\begin{tabular}{|c|c|c|}
\hline Variável & $\mathbf{n}$ & $\%$ \\
\hline \multicolumn{3}{|l|}{ Faixa etária (anos) } \\
\hline $40-50$ & 201 & 45,8 \\
\hline $51-60$ & 130 & 29,6 \\
\hline $61-69$ & 108 & 24,6 \\
\hline \multicolumn{3}{|l|}{ Raça/Cor } \\
\hline Branca & 356 & 81,1 \\
\hline Não branca & 83 & 18,9 \\
\hline \multicolumn{3}{|l|}{ Escolaridade } \\
\hline Analfabeta & 92 & 21,0 \\
\hline Primário completo/Ginasial incompleto & 149 & 33,9 \\
\hline Ginasial completo/Colegial incompleto & 64 & 14,6 \\
\hline Colegial completo/Superior incompleto & 100 & 22,8 \\
\hline Superior completo & 34 & 7,7 \\
\hline \multicolumn{3}{|l|}{ Classe econômica * } \\
\hline$A$ e $B$ & 112 & 25,5 \\
\hline C & 241 & 54,9 \\
\hline$D$ e $E$ & 86 & 19,6 \\
\hline \multicolumn{3}{|l|}{ Estado civil } \\
\hline Casada & 302 & 68,8 \\
\hline Solteira/Separada & 83 & 18,9 \\
\hline Viúva & 54 & 12,3 \\
\hline
\end{tabular}

* Variável baseada no Critério de Classificação Econômica Brasil da Associação Nacional de Empresas de Pesquisa 26.

aquela que apresenta as menores prevalências de realização de mamografia alguma vez na vida (valor de $\mathrm{p}=0$,0325) (Tabela 5).

Quando realizada a análise bruta, demonstrase que as mulheres que fizeram tratamento para a menopausa e já interromperam são que menos realizaram a mamografia $(\mathrm{p}=0,001)$ (Tabela 5).

\section{Associação entre as condutas preventivas do câncer de mama}

Somente não foi observada correlação entre o fato de a mulher ter realizado mamografia e possuir o hábito de examinar suas mamas em casa $(\mathrm{p}>0,05)$.

Das 64,5\% (283) mulheres que possuem o hábito de examinar as suas mamas em casa, a maioria $(75,3 \%)$ refere que suas mamas foram examinadas na última consulta ginecológica pelo médico ou enfermeiro. Isso leva a afirmar que as mulheres que têm o hábito de examinar as mamas em casa são aquelas que foram examinadas com maior frequência por profissionais de saúde na última consulta ginecológica ( $\mathrm{p}=0,001)$.

As mulheres que tiveram, na última consulta ginecológica, suas mamas examinadas pelo mé- dico ou enfermeiro são, em sua maioria, aquelas que também fizeram mamografia $(p=0,000)$.

\section{Discussão}

Dentre todos os fatores estudados na realização da prevenção secundária do câncer de mama, a classe econômica é o único fator em comum que interfere nas condutas preventivas, tanto no autoexame das mamas quanto no exame clínico das mamas e na mamografia.

Pesquisa de base populacional realizada em Pelotas (Rio Grande do Sul) observou associação diretamente proporcional entre o nível socioeconômico e a realização de condutas preventivas, ou seja, quanto mais elevado o nível socioeconômico, maior é também o número de consultas realizadas e, consequentemente, mais numerosos são os exames realizados, com maior oportunidade de um diagnóstico precoce ${ }^{9}$. Por sua vez, a história familiar de câncer de mama sugere um aumento no estímulo às práticas preventivas, apesar de somente $5 \%$ a $10 \%$ das mulheres com câncer possuírem familiares com a doença 11 . 
Tabela 2

Distribuição percentual das entrevistadas segundo realização do autoexame das mamas, exame clínico da mama e mamografia. Maringá, Paraná, Brasil, 2008.

\begin{tabular}{|c|c|c|}
\hline Variável & $\mathbf{n}$ & $\%$ \\
\hline \multicolumn{3}{|c|}{ Realização do autoexame das mamas } \\
\hline Sim & 283 & 64,5 \\
\hline Não & 156 & 35,5 \\
\hline \multicolumn{3}{|c|}{ Frequência do autoexame das mamas (vezes) } \\
\hline 1 & 7 & 2,5 \\
\hline 2 & 10 & 3,5 \\
\hline 3 & 8 & 2,8 \\
\hline 4 & 3 & 1,1 \\
\hline 5 ou mais & 217 & 76,7 \\
\hline Não sabe/Não lembra & 38 & 13,4 \\
\hline \multicolumn{3}{|l|}{ Exame clínico das mamas } \\
\hline Sim & 314 & 71,5 \\
\hline Não & 106 & 24,1 \\
\hline Não sabe/Não lembra & 8 & 1,8 \\
\hline \multicolumn{3}{|l|}{ Realização de mamografia } \\
\hline Sim & 347 & 79,0 \\
\hline Não & 92 & 21,0 \\
\hline \multicolumn{3}{|c|}{ Tempo de realização da mamografia (meses) } \\
\hline $1-6$ & 105 & 30,2 \\
\hline $7-12$ & 114 & 32,9 \\
\hline $13-24$ & 74 & 21,3 \\
\hline $25-360$ & 54 & 15,6 \\
\hline
\end{tabular}

Tabela 3

Distribuição percentual das entrevistadas segundo realização do autoexame das mamas e a relação com a classe econômica. Maringá, Paraná, Brasil, 2008.

\begin{tabular}{|c|c|c|c|c|}
\hline Variável & $\operatorname{Sim}(\%)$ & Valor de p & OR bruta (IC95\%) & $\begin{array}{l}\text { OR ajustada } \\
\text { (IC95\%) }\end{array}$ \\
\hline Classe econômica & & 0,0325 * & & \\
\hline B1 e B2 & $15(3)$ & & 1,000 & 1,000 \\
\hline C & $174(40)$ & & $1,718(1,153-2,558)$ & $1,766(1,178-2,648)$ \\
\hline$D$ e $E$ & $158(36)$ & & $1,111(0,409-3,021)$ & $1,452(0,525-4,013)$ \\
\hline
\end{tabular}

IC95\%: intervalo de 95\% de confiança; OR: odds ratio.

* Qui-quadrado.

Por meio da mamografia, é possível reduzir as taxas de mortalidade por câncer de mama, e um estudo randomizado com mulheres entre 40-70 anos na Europa e na América do Norte mostrou os benefícios desse exame. Em mulheres que realizaram a mamografia como prevenção secundária do câncer de mama, observou- se uma diminuição de $63 \%$ de mortes por câncer de mama. Nos Estados Unidos, apesar de uma incidência crescente do câncer de mama, as taxas de mortalidade têm decaído entre as mulheres que realizam mamografia, o que pode ser resultante de uma melhora da qualidade técnica da mamografia devido aos avanços dos 
Tabela 4

Distribuição percentual das entrevistadas segundo realização do exame clínico das mamas e a relação entre classe econômica, escolaridade e terapia de reposição hormonal. Maringá, Paraná, Brasil, 2008.

\begin{tabular}{|c|c|c|c|c|}
\hline Variável & $\operatorname{Sim}(\%)$ & Valor de $p$ & OR bruta (IC95\%) & OR ajustada (IC95\%) \\
\hline Classe econômica & & 0,0044 * & & \\
\hline B1 e B2 & $18(4)$ & & 1,000 & 1,000 \\
\hline C & $157(37)$ & & $1,456(0,931-2,275)$ & $1,204(0,730-1,985)$ \\
\hline$D e E$ & $140(33)$ & & ** & $\star \star$ \\
\hline Escolaridade & & 0,0005 * & & \\
\hline Analfabeta & $51(12)$ & & $0,440(0,263-0,734)$ & $0,477(0,244-0,817)$ \\
\hline Primário completo & $104(25)$ & & 1,000 & 1,000 \\
\hline Ginasial completo & $51(12)$ & & $1,869(0,912-3,838)$ & $1,547(0,715-3,347)$ \\
\hline Colegial completo & $75(18)$ & & $1,137(0,670-1,932)$ & $1,016(0,559-1,846)$ \\
\hline Superior completo & $33(8)$ & & $\star \star \star$ & $\star \star \star$ \\
\hline Reposição hormonal & & 0,0491 * & & \\
\hline Não, nunca fez & $211(50)$ & & 1,000 & 1,000 \\
\hline Está fazendo & $31(8)$ & & $0,667(0,345-1,290)$ & $0,767(0,386-1,523)$ \\
\hline Fez, mas já parou & $71(17)$ & & $2,099(1,109-3,972)$ & $2,476(1,264-4,849)$ \\
\hline
\end{tabular}

IC95\%: intervalo de 95\% de confiança.

* Qui-quadrado;

** Não foi possível estimar a odds ratio (OR) para essa categoria (não possui casos suficientes para estimar);

*** Estimativa imprecisa, foi retirada do modelo.

Tabela 5

Distribuição percentual das entrevistadas segundo realização de mamografia e a relação entre faixa etária, raça, classe econômica e terapia de reposição hormonal. Maringá, Paraná, Brasil, 2008.

\begin{tabular}{|c|c|c|c|c|}
\hline Variável & $\operatorname{Sim}(\%)$ & Valor de p & OR bruta (IC95\%) & OR ajustada (IC95\%) \\
\hline Faixa etária (anos) & & $<0,0001$ * & & \\
\hline $40-50$ & $142(32)$ & & 1,000 & 1,000 \\
\hline $51-60$ & $118(27)$ & & $0,290(0,152-0,553)$ & $0,268(0,131-0,548)$ \\
\hline $61-69$ & $87(20)$ & & $0,894(0,519-1,545)$ & $0,581(0,310-1,089)$ \\
\hline Raça & & 0,0479 * & & \\
\hline Branca & $288(66)$ & & 1,000 & 1,000 \\
\hline Não branca & $59(13)$ & & $1,717(0,997-2,956)$ & $1,705(0,954-3,045)$ \\
\hline Classe econômica & & 0,0325 * & & \\
\hline B1 e B2 & $15(3)$ & & 1,000 & 1,000 \\
\hline C & $174(40)$ & & $0,559(0,348-0,900)$ & $0,452(0,269-0,758)$ \\
\hline$D$ e $E$ & $158(36)$ & & $0,744(0,211-2,626)$ & $0,499(0,131-1,903)$ \\
\hline Reposição hormonal & & $<0,0001 *$ & & \\
\hline Não, nunca fez & $224(51)$ & & 1,000 & 1,000 \\
\hline Está fazendo & $45(10)$ & & $0,225(0,068-0,743)$ & $0,258(0,077-0,870)$ \\
\hline Fez, mas já parou & $78(18)$ & & $0,379(0,182-0,788)$ & $0,452(0,201-1,019)$ \\
\hline
\end{tabular}

IC95\%: intervalo de 95\% de confiança.

* Qui-quadrado. 
equipamentos utilizados. Os fatores associados ao aumento de detecção precoce do câncer de mama pela mamografia foram: técnica melhorada, realização de duas mamografias por mama, intervalos anuais entre um exame e outro e interpretação melhorada 12 .

Em pesquisa realizada com mulheres brasileiras por meio da Pesquisa Nacional por Amostra de Domicílios (PNAD), constatou-se que os fatores preditivos para a realização da mamografia são referentes à distribuição etária (40-59 anos), consulta médica no último ano, morar em zona urbana, renda elevada e possuir plano de saúde 13. Em outro estudo, observou-se que a cobertura da mamografia ainda é baixa entre as mulheres brasileiras, principalmente entre as mais velhas, e que há profundas desigualdades associadas à sua realização ${ }^{14}$. Em outro estudo, encontrou-se que a escolaridade, o estado civil, a profissão e a renda da mulher são fatores que interferem na prevalência da realização de mamografia 15.

No Município de Campinas (São Paulo), em 2001, uma pesquisa demonstrou que a principal barreira à realização da mamografia, em 81,8\% dos casos, foi a falta de solicitação por parte dos médicos dos centros de saúde em estudo. Nesse mesmo estudo, constatou-se que a prática adequada da mamografia associou-se com o trabalho fora de casa e renda familiar igual ou superior a cinco salários-mínimos 16 .

Vindo ao encontro de diversos estudos, na presente pesquisa, constatou-se que as mulheres que menos realizaram a mamografia foram as de classe socioeconômica D e E e as que fizeram tratamento para a menopausa e já interromperam.

Referente ao exame clínico das mamas, em São Leopoldo (Rio Grande do Sul), estudo de base populacional demonstrou que, dentre todas as variáveis estudadas, somente a classe econômica estava associada com a não realização de exame médico das mamas. Entre as mulheres que haviam feito consulta médica no ano da realização do estudo, a cor da pele foi determinante para a realização do exame, resultando numa maior possibilidade de as mulheres brancas serem examinadas 17 .

Fato que foi observado em outra pesquisa seria a relação da obesidade com a menor prevalência da prática preventiva, visto que a obesidade é referida como fator de risco para o desenvolvimento do câncer de mama 18. Entretanto, não foi observado, neste estudo, correlação entre o índice de massa corpórea (IMC) e a realização da prevenção secundária do câncer de mama.

Estudos confirmam alguns fatores associados a maiores prevalências de realização da prevenção secundária do câncer de mama, entre os quais, encontram-se bom nível socioeconômico, história familiar de câncer de mama e história pessoal de biopsia 19 .

Estudos sobre fatores socioeconômicos e a prática de prevenção de câncer de mama relatam que mulheres de maior nível educacional e renda são as que mais aderem e as que detêm maior conhecimento sobre o autoexame das mamas. Essa prática constitui um dos fatores essenciais à prevenção secundária do câncer de mama e resulta em um diagnóstico precoce e, consequentemente, na diminuição da incidência de mortalidade. As mulheres de baixo estrato social, educacional e informacional são as que mais necessitam ser orientadas para a prática do autoexame da mama. Com isso, observa-se também que as mulheres que retardam a consulta ao médico especializado e procuram assistência em estágios avançados da doença são mulheres mais velhas e de classe social e educacional mais baixas 20 .

Pesquisa realizada com mulheres de 40-69 anos diagnosticou, como um dos principais fatores associados a maiores prevalências das condutas na prevenção secundária do câncer de mama, pertencer às classes sociais mais altas e, consequentemente, possuir um maior nível de escolaridade. Mais uma vez, o nível socioeconômico parece ser fator que mais favorece o acesso à consulta ginecológica e a adoção das demais condutas de prevenção secundária do câncer de mama, o que contribui para a diminuição da incidência de mortalidade 9 .

De forma inversa, em outro estudo, observouse que as mulheres de classes sociais mais baixas relataram menor acesso aos serviços de saúde e a exames clínicos das mamas. As mulheres não brancas também relataram esse resultado 17 .

O presente estudo revela que um dos fatores em comum para a realização das condutas preventivas foi a classe econômica das entrevistadas. As mulheres que pertencem às camadas sociais mais baixas têm menor oferta de mamografia e menos exames clínicos das mamas realizados por médico ou enfermeiro. Ademais, essa mesma população realiza menos o autoexame das mamas.

No Município de Botucatu (São Paulo), em um estudo com mulheres entre 30 e 84 anos, identificou-se que as idosas apresentavam uma taxa menor de oportunidades de diagnóstico precoce. Elas eram menos informadas sobre a periodicidade correta do autoexame das mamas e relataram uma menor frequência de mamografia e de exame clínico das mamas do que as mulheres jovens. Constatou-se também que o grau de escolaridade possui significância estatística, sendo que mulheres com mais anos de estudo 
obtiveram melhores oportunidades do que as com pouco estudo ${ }^{21}$.

Outro estudo de base populacional, com mulheres de 20-69 anos, encontrou que 48,9\% das entrevistadas tiveram suas mamas examinadas pelo médico, sendo que a classe social, cor da pele, frequência de consulta médica e realização de exame citopatológico estavam associadas a essa prática de conduta preventiva. $\mathrm{O}$ mesmo estudo afirma que há desigualdade na atenção à saúde e falta de integralidade na assistência à mulher 22 .

A deficiência no sistema de saúde pode resultar numa iniquidade em relação às ações de prevenção do câncer de mama, como as que foram citadas acima.

Verificou-se, ainda, em outra pesquisa, que mulheres que possuíam até quatro anos de estudo sabiam menos sobre a periodicidade do autoexame das mamas do que as mulheres que estudaram nove anos ou mais. No estudo acima, não foi encontrada relação estatisticamente significativa entre o conhecimento da periodicidade recomendada do autoexame das mamas e algumas variáveis, destacando-se, entre essas, a profissão, antecedentes familiares de câncer de mama e ter recebido orientação sobre a prevenção do câncer de mama e sobre como fazer o autoexame das mamas 21 .

No presente estudo, dentre todos os fatores estudados, foi encontrada, como fatores associados à realização do autoexame das mamas, somente a classe econômica da mulher. Assim, como no estudo de Molina et al. ${ }^{21}$, não se observou correlação entre o autoexame das mamas e antecedentes familiares de câncer de mama.

Estudo com 2.073 pacientes obteve, como resultado, que $75 \%$ das mulheres conheciam o autoexame das mamas, e $51 \%$ o praticavam. A realização do autoexame das mamas foi influenciada positivamente pelos fatores: ser dona de casa, possuir 30 anos ou mais, possuir maior escolaridade e renda superior a dois saláriosmínimos. Infere-se que as mulheres pertencentes à camada da população mais carente são as que possuem menos conhecimento sobre o autoexame das mamas e consequentemente o realizam menos 23 .

Observou-se que as mulheres com nove anos de estudo ou mais foram expostas com maior frequência ao exame clínico das mamas do que as que estudaram por até quatro anos. A idade demonstrou também estar relacionada com maior oportunidade de receber o exame clínico das mamas, sendo que as mulheres mais jovens foram examinadas em maior número do que as mais velhas. Porém não foi encontrada correlação com número de filhos da mulher e a realização do exame clínico das mamas 17. Em compa- ração com a pesquisa acima, neste estudo, não houve correlação com a escolaridade da mulher, somente com a classe econômica.

Quanto à mamografia, constata-se uma diferença estatisticamente significativa entre a escolaridade e a solicitação de mamografia, sendo que as mulheres com nove anos ou mais de estudo referiram uma frequência maior de mamografia do que as que estudaram por até quatro anos 21 .

A maioria dos programas de prevenção do câncer de mama recomenda, principalmente em locais onde há muitos casos de diagnóstico tardio, a realização do autoexame como uma das formas de detecção precoce. Na literatura, é enfatizado que o autoexame das mamas é subutilizado por mulheres pertencentes a classes sociais mais elevadas, ao passo que a mamografia, a principal arma no diagnóstico precoce do câncer de mama, deixa de fora significativa parcela da população feminina por não ter acesso a esse procedimento mais sofisticado 24 . Cumpre ressaltar a importância da realização do autoexame das mamas em populações que têm dificuldade de acesso à mamografia.

Pesquisa realizada com mulheres acima de 65 anos constatou que o exame clínico das mamas era a prática mais usada por essas mulheres, seguida da mamografia e, distantemente, em terceiro lugar, do autoexame das mamas. As razões mais citadas para a não realização de mamografia foram não apresentar sintomas, negligência, esquecimento ou não ter tido indicação de um especialista. Não houve diferença estatisticamente significativa entre o conhecimento e as práticas de detecção precoce. Ao contrário, acreditar nas medidas preventivas teve um impacto na sua realização. Nesse mesmo estudo, também foi demonstrado que a idade está associada com a execução do autoexame das mamas uma a duas vezes por mês 25 .

O mesmo autor sustenta que o nível social mais elevado associa-se à realização do autoexame das mamas e a nunca ter realizado mamografia. O grau de instrução está correlacionado positivamente à realização de mamografia e ter realizado mamografia nos dois últimos anos anteriores à entrevista. Os fatores encontrados referentes à realização da mamografia nos dois últimos anos foram a indicação de um especialista, possuir automóvel e receber orientação de um profissional de saúde após a menopausa ou câncer de mama. A referência de um médico foi o fator mais importante para a realização da mamografia 25.

Não foi observada diferença significativa entre o fato de a mulher ter feito mamografia e o hábito de examinar suas mamas em casa. A re- 
alização do autoexame das mamas tem associação positiva com o exame clínico das mamas. $\mathrm{O}$ mesmo ocorre entre o exame clínico das mamas e a mamografia.

Nos estudos de Sclowitz et al. 9, observa-se resultado semelhante, pois não se observou associação entre "hábito de realizar autoexame das mamas" e "realização de mamografia na vida".

\section{Conclusão}

Os dados dessa pesquisa mostram que a maior parte das mulheres estudadas pertence à raça branca $(81,1 \%)$ e são da classe econômica classificada como C. Poucas $(7,7 \%)$ possuíam o ensino superior completo.

Os fatores encontrados associados à realização do autoexame das mamas, exame clínico das mamas e mamografia foram a faixa etária, escolaridade, classe econômica e realização de tratamento para menopausa. Observou-se correlação entre exame clínico das mamas e autoexame das mamas e também entre a realização do exame clínico das mamas e a mamografia.

O fator classe econômica influencia significativamente as práticas de prevenção secundária do câncer de mama, de modo que quanto mais alto o nível socioeconômico da mulher, maior a prevalência de realização dessas condutas. Esses dados demonstram que o Município de Maringá, em relação às práticas preventivas do câncer de mama, encontra as mesmas barreiras observadas em outros municípios brasileiros.

A orientação quanto à realização do autoexame das mamas, exame clínico das mamas e mamografia deve estar ao alcance de todas as mulheres, independente de sua classe socioeconômica. Essa mudança deve ser realizada não só internamente, com a conscientização das mulheres sobre a importância dessas medidas preventivas, mas externamente também, oferecendo-se, de maneira igualitária, oportunidades de diagnóstico precoce a todas as mulheres na faixa etária de maior risco de desenvolver o câncer de mama.

Outra questão que deve ser discutida e influencia nas possibilidades de tratamento e na cura do câncer de mama é o tempo de espera para realização de exames e a localização do domicílio dessas mulheres, o que pode indicar problemas no acesso ao serviço de saúde.

Ressaltam-se as limitações desse estudo em relação à metodologia utilizada e a necessidade de novas questões investigativas para compreender melhor o fenômeno do aumento de casos e da mortalidade por câncer de mama.

O câncer de mama, quando diagnosticado precocemente, apresenta grandes chances de cura, o que pode evitar muitas mortes desnecessárias, o sofrimento causado pelo tratamento e incapacidades provenientes da doença.

\section{Resumo}

Este estudo analisou a prevalência e os fatores associados à realização da prevenção secundária do câncer de mama em mulheres de 40-69 anos do Município de Maringá, Paraná, Brasil. Realizou-se um estudo exploratório de corte transversal, tipo inquérito populacional, no Município de Maringá. A prevalência observada de realização do autoexame das mamas foi de 64,5\%, do exame clínico das mamas, 71,5\%, e de mamografia, $79 \%$. Os fatores que influenciaram na realização dessas ações foram: idade, escolaridade, raça, classe econômica, religião e realização de terapia hormonal. A classe econômica e a escolaridade influenciaram significativamente na realização das práticas preventivas, ou seja, quanto maior o nível socioeconômico, maior a realização. A orientação e realização das práticas preventivas devem estar ao alcance de todas as mulheres na faixa etária de maior risco, diminuindo, dessa maneira, o diagnóstico tardio e mortes desnecessárias e aumentando as chances de cura do câncer de mama.

Neoplasias de Mama; Prevenção Secundária; Diagnóstico Precoce; Prevalência 


\section{Colaboradores}

J. C. Matos participou de todas as etapas do trabalho: concepção do estudo, trabalho de campo, interpretação dos dados, discussão e elaboração do artigo final. S. M. Pelloso contribuiu na elaboração e organização do estudo, na análise dos dados e na revisão crítica do artigo. M. D. B. Carvalho contribuiu na concepção e projeto, revisão crítica do conteúdo intelectual e aprovação final da versão a ser publicada.

\section{Referências}

1. Coordenação de Prevenção e Vigilância, Instituto Nacional de Câncer. Controle do câncer de mama: documento de consenso. Rio de Janeiro: Instituto Nacional de Câncer; 2004.

2. Instituto Nacional de Câncer. Estimativas da incidência e mortalidade por câncer no Brasil 2006. Rio de Janeiro: Instituto Nacional de Câncer; 2006.

3. Instituto Nacional de Câncer. Programa nacional de controle do câncer do colo do útero e da mama - viva mulher. http://www.inca.gov.br/conteudo_ view.asp?id=140 (acessado em 12/Mar/2010).

4. Instituto Nacional de Câncer. Incidência do câncer no Brasil: estimativa 2010. http://www.inca.gov. br/estimativa/2010 (acessado em 09/Jan/2011).

5. Prefeitura do Município de Maringá. Lançamento da Campanha contra o Câncer de Mama, 2007. http://www2.maringa.pr.gov.br/site/index.php?se ssao=b76ca7228055b7\&id=5239 (acessado em 24/ Mai/2007).

6. Pinho VFS, Coutinho ESF. Variáveis associadas ao câncer de mama em usuárias de unidades básicas de saúde. Cad Saúde Pública 2007; 23:1061-9.
7. Godinho ER, Koch HA. O perfil da mulher que se submete à mamografia em Goiânia: uma contribuição a Bases para um Programa de Detecção Precoce do Câncer de Mama. Radiol Bras 2002; 35:139-45.

8. Instituto Nacional de Câncer. Detecção precoce do câncer de mama. http://www.inca.gov.br/conteu do_view.asp?id=1932 (acessado em 23/Abr/2010).

9. Sclowitz ML, Menezes AMB, Gigante DP, Tessaro S. Condutas na prevenção secundária do câncer de mama e fatores associados. Rev Saúde Pública 2005; 39:340-9.

10. Matos JC. Prevalência e fatores associados à prevenção secundária do câncer de mama em Maringá-PR [Dissertação de Mestrado]. Maringá: Universidade Estadual de Maringá; 2008.

11. Pharoah PD, Day NE, Duffy S, Easton DF, Ponder BAJ. Family history and the risk of breast cancer: a systematic review and meta-analysis. Int J Cancer 1997; 71:800-9. 
12. Feig SA. Screening mammography: a successful public health initiative. Rev Panam Salud Pública 2006; 20:125-33.

13. Novaes HMD, Braga PE, Schout D. Fatores associados à realização de exames preventivos para câncer nas mulheres brasileiras, PNAD 2003. Ciênc Saúde Coletiva 2006; 11:1023-35.

14. Lima-Costa MF, Matos DL. Prevalência e fatores associados à realização da mamografia na faixa etária de 50-69 anos: um estudo baseado na Pesquisa Nacional por Amostra de Domicílios (2003). Cad Saúde Pública 2007; 23:1665-73.

15. Bim CR. Avaliação da qualidade de saúde da mulher em Guarapuava - PR [Dissertação de Mestrado]. Maringá: Universidade Estadual de Maringá; 2007.

16. Marinho LAB, Cecatti JG, Osis MJD, Gurgel MSC. Knowledge, attitude and practice of mammography among women users of public health services. Rev Saúde Pública 2008; 42:576-82.

17. Dias-da-Costa JS, Olinto MTA, Bassani D, Marchionatti CRE, Bairros FS, Oliveira MLP, et al. Desigualdades na realização do exame clínico de mama em São Leopoldo, Rio Grande do Sul, Brasil. Cad Saúde Pública 2007; 23:1603-12.

18. Wee CC, McCarthy EP, Davis RB, Phillips RS. Screening for cervical and breast cancer: is obesity an unrecognized barrier to preventive care? Ann Intern Med 2000; 132:697-704.

19. Boyle P. Current situation of screening for cancer. Ann Oncol 2002; 13:189-98.
20. Gonçalves SCM, Dias MR. A prática do autoexame da mama em mulheres de baixa renda: um estudo de crenças. Estud Psicol (Natal) 1999; 4:141-59.

21. Molina L, Dalben I, Luca LA. Análise das oportunidades de diagnóstico precoce para as neoplasias malignas de mama. Rev Assoc Med Bras (1992) 2003; 49:185-90.

22. Costa JSD, Olinto MTA, Gigante DP, Menezes AMB, Macedo S, Fuchs SC. Coverage of physical breast examination to prevent breast cancer: a population-based study in Pelotas, RS. Rev Bras Epidemiol 2003; 6:39-48.

23. Freitas Júnior R, Koifman S, Santos NRM, Nunes MOA, Melo GG, Ribeiro ACG, et al. Conhecimento e prática do autoexame de mama. Rev Assoc Med Bras (1992) 2006; 52:97-109.

24. Marinho LAB, Costa-Gurgel MS, Cecatti JG, Osis MJD. O papel do autoexame mamário e da mamografia no diagnóstico precoce do câncer de mama. Rev Cienc Med (Campinas) 2002; 11:167-80.

25. Ayéndez M, Suaréz-Peréz E, Vázquez MO, VélezAlmodóvar H, Nazario CM. Knowledge and beliefs of breast cancer among elderly women in Puerto Rico. P R Health Sci J 2001; 20:351-9.

26. Associação Nacional de Empresas de Pesquisas. Critério de classificação econômica Brasil. http:// www.datavale-sp.com.br/CCEB.pdf (acessado em 14/Jan/2010).

Recebido em 12/Jul/2010

Versão final reapresentada em 20/Fev/2011

Aprovado em 11/Mar/2011 